\title{
The Basic Helix-Loop-Helix-PAS Protein MOP9 Is a Brain-Specific Heterodimeric Partner of Circadian and Hypoxia Factors
}

\author{
John B. Hogenesch, ${ }^{1}$ Yi-Zhong Gu, ${ }^{1}$ Susan M. Moran, ${ }^{1}$ Kazuhiro Shimomura, ${ }^{2}$ Laurel A. Radcliffe, ${ }^{2}$ \\ Joseph S. Takahashi, ${ }^{2}$ and Christopher A. Bradfield ${ }^{1}$ \\ 1 The McArdle Laboratory for Cancer Research, University of Wisconsin Medical School, Madison, Wisconsin 53706, and \\ 2The Howard Hughes Medical Institute, Department of Neurobiology and Physiology, Northwestern University, Evanston, \\ Illinois 60208-3520
}

\begin{abstract}
PAS (PER, ARNT, SIM) proteins play important roles in adaptation to low atmospheric and cellular oxygen levels, exposure to certain environmental pollutants, and diurnal oscillations in light and temperature. In an attempt to better understand how organisms sense environmental changes, we have characterized a novel member of the PAS superfamily, MOP9 (member of PAS superfamily), that maps to human chromosome 12p11.2211.23. This protein displays significant homology to the Drosophila circadian factor CYCLE and its putative mammalian ortholog MOP3/bMAL1. Like its homologs, MOP9 forms a tran-
\end{abstract}

Members of the PAS (PER, ARNT, SIM) superfamily of eukaryotic transcription factors regulate biological responses to light, oxygen, and polycyclic aromatic hydrocarbons (Taylor and Zhulin, 1999). These proteins are distinguished by an $\sim 275$ amino acid motif termed the PAS domain, for PER, ARNT, and SIM homology domain (Reddy et al., 1986; Crews et al., 1988; Hoffman et al., 1991). The PAS domain functions as a dimerization surface for interactions between PAS proteins and as a docking site for cellular chaperones (Huang et al., 1993; Perdew and Bradfield 1996; Carver et al., 1998). In the case of the aryl hydrocarbon receptor (AHR), the PAS domain also functions as a binding surface for structurally related agonists (Burbach et al., 1992). The majority of PAS proteins also harbor a basic helixloop-helix (bHLH) domain immediately N-terminal to PAS. This domain supports dimerization and provides a basic $\alpha$-helix required for specific DNA contacts within target enhancer elements (Kadesch, 1993).

Members of the bHLH-PAS superfamily can be classified along phylogenetic and functional lines (Gu et al., 2000). Members of the $\alpha$-class often function as "sensors" of environmental stimuli. The mammalian $\beta$-class members typically act as general partners for a broad array of $\alpha$-class molecules. For example, $\alpha$-class proteins such as the AHR are activated by the binding of polycyclic aromatic hydrocarbons and dimerize with $\beta$-class molecules such as ARNT (Burbach et al., 1992; Reisz-Porszasz et al., 1994). Other $\alpha$-class proteins such as HIF1 $\alpha$, HIF2 $\alpha$, and HIF3 $\alpha$ are upregulated by low oxygen tension and can also dimerize with $\beta$-class molecules such as ARNT, ARNT2 and MOP3 (member of PAS superfamily) (Wang et al., 1995; Pugh et al., 1997; Gu et

\footnotetext{
Received Feb. 22, 2000; revised Sept. 10, 2000; accepted April 11, 2000.

This work was supported by The Burroughs Wellcome Foundation and National Institutes of Health Grants P30-CA07175, ES05703, and GM08061. J.S.T. is an Investigator and K.S. is an Associate in the Howard Hughes Medical Institute. The GenBank accession numbers for the proteins in this paper are AF231338 and AF231339.

Correspondence should be addressed to Christopher A. Bradfield, McArdle Laboratory for Cancer Research, 1400 University Avenue, Madison, WI 53706. E-mail: bradfield@oncology.wisc.edu.

Copyright (C) 2000 Society for Neuroscience $0270-6474 / 00 / 200001-05 \$ 15.00 / 0$
}

scriptionally active heterodimer with the circadian CLOCK protein, the structurally related MOP4, and hypoxia-inducible factors, such as HIF1 $\alpha$. In a manner consistent with its role as a biologically relevant partner of these proteins, MOP9 is coexpressed in regions of the brain such as the thalamus, hypothalamus, and amygdala. Importantly, MOP9 is coexpressed with CLOCK in the suprachiasmatic nucleus, the site of the master circadian oscillator in mammals.

Key words: MOP9; bHLH-PAS; circadian; hypoxia; transcription factor; heterodimer; SCN

al., 1998; Hogenesch et al., 1998). Members of a third class we designate as the $\gamma$-class, have been shown to function as coactivator molecules. These proteins, SRC1, TIF2, and RAC3, interact with members of the nuclear hormone receptor superfamily and link them to the CBP/p300 complex (Hanstein et al., 1996; Chen and Li, 1998).

Recently, molecular and genetic data have provided strong evidence that a bHLH-PAS $\alpha$ - $\beta$ heterodimer is central to the maintenance of circadian rhythms in species found throughout the animal kingdom (Dunlap, 1999). In mammalian systems, a combination of genetic and biochemical evidence points to the importance of a dimer of CLOCK and MOP3 (also known as bMAL1) in regulating rhythmicity (Gekakis et al., 1998; Hogenesch et al., 1998). In Drosophila, compelling genetic evidence supports a role for putative orthologs of these two proteins denoted CLOCK and CYCLE, respectively (Allada et al., 1998; Rutila et al., 1998). In this report, we provide evidence that regulation of the mammalian circadian rhythm may be more complicated than previously thought. In this regard, we present a novel homolog of MOP3 that we designate MOP9. The MOP9 protein meets the criteria of a biologically relevant partner of CLOCK in that it forms transcriptionally active complexes with CLOCK and is regionally coexpressed in the suprachiasmatic nucleus, the sight of the central circadian pacemaker in mammals. In addition, like MOP3, MOP9 can also interact with HIF1 $\alpha$,

This article is published in The Journal of Neuroscience, Rapid Communications Section, which publishes brief, peer-reviewed papers online, not in print. Rapid Communications are posted online approximately one month earlier than they would appear if printed. They are listed in the Table of Contents of the next open issue of JNeurosci. Cite this article as: JNeurosci, 2000, 0: RC83 (1-5). The publication date is the date of posting online at www.jneurosci.org.

http://www.jneurosci.org/cgi/content/full/4296 
providing a potential physiological link between circadian rhythmicity and cellular oxygen status.

\section{MATERIALS AND METHODS}

Cloning of MOP9. The Drosophila CYCLE sequence was used to search the mammalian GenBank NR database using the tblastn algorithm (Altschul et al., 1990). This search uncovered an expressed sequence tag (EST), GBAA577389, with significant homology to human MOP3. Oligonucleotides based on this sequence were designed and used to amplify the $5^{\prime}$ end of this gene using the Marathon Ready cDNA system and modified human brain cDNA as template (Clontech, Palo Alto, CA). Oligonucleotides designed against the human MOP9 cDNA were used to amplify a fragment of the mouse cDNA using the PCR and mouse Marathon Ready cDNA (Clontech). Finally, the PCR was used to amplify the entire open reading frame of the human MOP9. This fragment was then cloned into pTarget (PL1480) for expression in mammalian cells (Promega, Madison, WI)

Expression of MOP9. A $1.5 \mathrm{~kb}$ PCR fragment of MOP9 was random primed (Amersham, Cleveland, $\mathrm{OH}$ ) and used as a probe in Northern blot analysis of various human tissues. Multiple-tissue Northern blots from fetal sources and two adult brain region blots were screened (Clontech) (Hogenesch et al., 1997). As a hybridization control, a human actin probe was subsequently used to screen both the multiple tissue and brain-specific Northern blots. For in situ analysis of MOP9 mRNA in mouse brain, a mouse cDNA probe was isolated by the PCR using mouse brain cDNA as template. The T3 and T7 primer sites were introduced on the $5^{\prime}$ and $3^{\prime}$ ends of the probe by PCR and used to prepare the riboprobe. The in situ analysis was performed on 17-week-old male mice, $[(\mathrm{BALB} / \mathrm{cJ} \times \mathrm{C} 57 \mathrm{BL} / 6 \mathrm{~J}) \mathrm{F} 1 \times \mathrm{C} 57 \mathrm{BL} / 6 \mathrm{~J}] \mathrm{N} 2$, as previously described (Sangoram et al., 1998).

Functional analysis. Transient transfection experiments were performed in Hep3B cells, and extracts were analyzed for luciferase and $\beta$-galactosidase activity as previously described (Hogenesch et al., 1997). Expression constructs harboring MOP3 (PL833), MOP4 (PL834), CLOCK (PL1386), HIF1 $\alpha$ (PL611), a reporter construct containing three M34 elements (sequence, 5' GGACACGTGACC $3^{\prime}$ ) upstream of luciferase (PL881), and a reporter construct harboring six hypoxia response elements (HREs; GGATACGTGACC) upstream of luciferase (PL949), have been previously described (Hogenesch et al., 1997; Hogenesch et al., 1998). Briefly, mammalian expression plasmids expressing hCLOCK, hMOP4, hMOP3, or hMOP9 were transfected with the MOP3-MOP4-bound responsive enhancer element (M34RE)-driven luciferase reporter in Hep3B cells. In addition, MOP9, ARNT, HIF1 $\alpha$, and a luciferase reporter driven by six HREs were transfected in Hep3B cells in the presence or absence of cobalt chloride (to mimic hypoxia). Transfections were performed using LipofectAMINE according to manufacturer's instructions (Life Technologies, Gaithersburg, MD). In all experiments, a $\beta$-galactosidase expression plasmid was cotransfected to control for transfection efficiency. After transfection, cells were incubated for $20 \mathrm{hr}$ before harvest, and luciferase and $\beta$-galactosidase activities were determined using a luciferase assay (Promega) and $\beta$-galactosidase assay (TROPIX, Bedford, MA), respectively.

Chromosomal localization of MOP9. PCR primers were designed against the region corresponding to the bHLH of the human MOP9 cDNA, and a fragment was amplified. This fragment was used to screen pooled BAC libraries, and a clone harboring the MOP9 structural gene was identified. Using the cloned bacterial artificial chromosome (BAC), fluorescence in situ hybridization (FISH) was performed on metaphase spreads as previously described (Genome Systems, St. Louis, MO) (Gu et al., 1998).

\section{RESULTS}

In a search of the GenBank human EST subset, a clone, GBAA577389, was identified that displayed significant homology to hMOP3 (blastx expect scores, $<4 \times 10^{-8}$ ) (Altschul et al., 1990). Sequence analysis of this EST suggested that the open reading frame was incomplete at the $5^{\prime}$ end. Therefore, the PCR was used to amplify the $5^{\prime}$ portion of the clone from a pool of human brain cDNA. In the process of this amplification, we identified a smaller product. Subsequent sequence analysis revealed that this smaller product was an in-frame splice variant of hMOP9, splicing out a short region of its $5^{\prime}$ end near the bHLH domain (Fig. $1 A$ ).

Sequence comparisons with the bHLH-PAS superfamily revealed that the hMOP9 amino acid sequence shares 72 and $74 \%$ identity with hMOP3 and dCYCLE, respectively, in the bHLH domain (Fig. 1A). In the PAS domain, hMOP9 shares 62 and $46 \%$ identity with hMOP3 and dCYCLE, respectively, whereas in the A and B repeats, the homology is higher, 80 and 68 and 77 and 57,

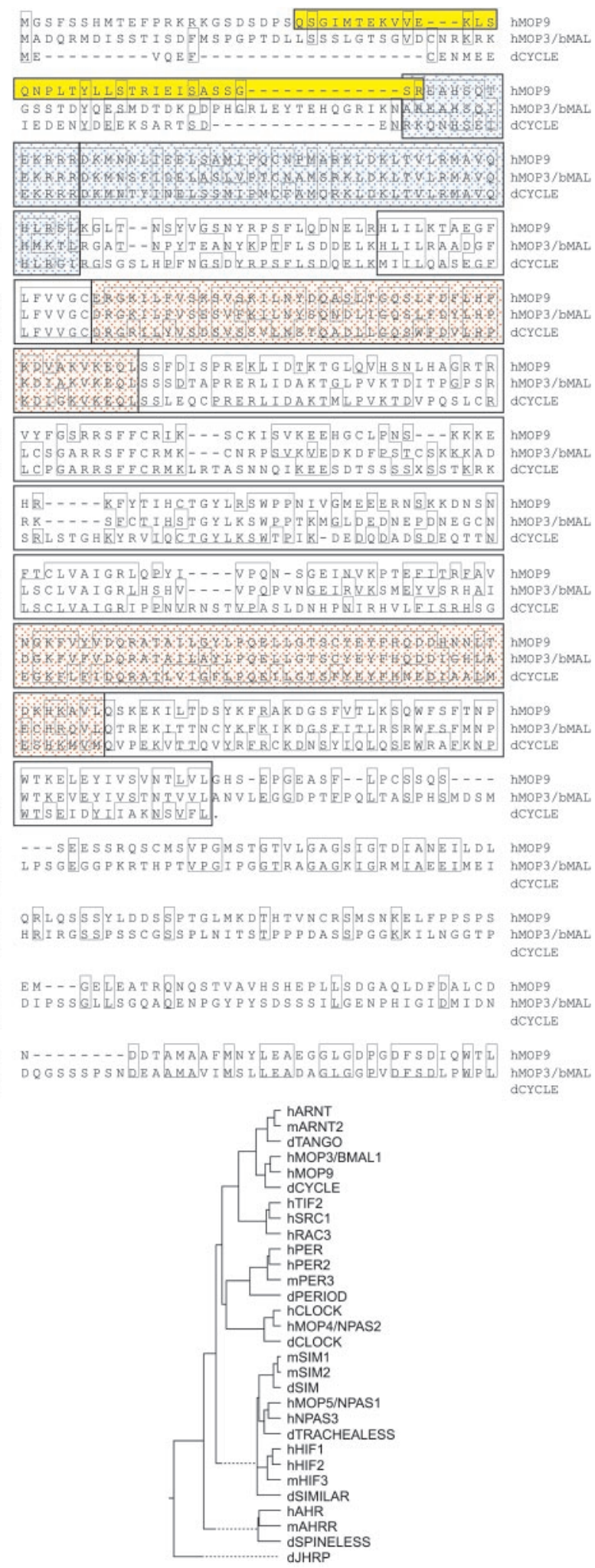

Figure 1. A, Primary amino acid sequence alignment. A Clustal alignment was performed with hMOP9, hMOP3, and CYCLE using the following parameters: gap penalty, 10; gap length penalty, 10. The bHLH domain is boxed in blue; the PAS domain is boxed; and the $\mathrm{A}$ and the $\mathrm{B}$ domains are red. The predicted amino acids removed by the splice variation of the MOP9 message are boxed in yellow. B, Phylogenetic analysis of the Drosophila and vertebrate bHLH-PAS members. $h, m, d$, Protein sequences used in the phylogeny from human, mouse, and Drosophila, respectively. 


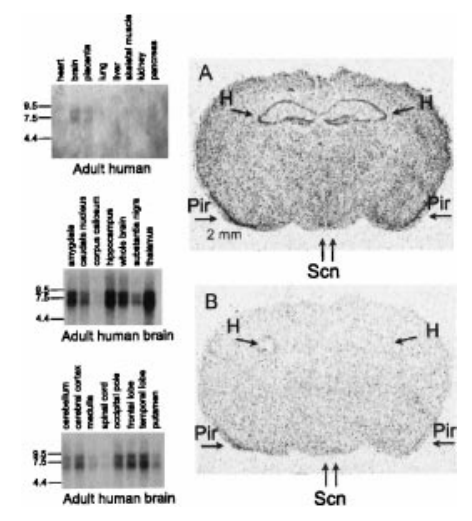

Figure 2. Expression analyses. Left, Northern blots containing organand brain-specific mRNAs were probed with a fragment of the MOP9 cDNA. These blots were all reprobed with an actin control probe to assure similar sample loading (data not shown). Right, In situ analysis of MOP9. Sense and antisense riboprobes were created using a fragment of the mouse MOP9 cDNA. Hybridization with the antisense probe $(A)$ revealed specific signal in the $\mathrm{SCN}$, hippocampus, and piriform cortex, whereas the sense probe $(B)$ did not produce a significant signal in these regions. The suprachiasmatic nucleus $(S c n)$, hippocampus $(H)$, and piriform cortex (Pir) are indicated by arrows.

respectively (Fig. 1A). A phylogenetic comparison among other members of the bHLH-PAS family revealed that MOP9 is most homologous to MOP3 and CYCLE (Fig. 1B). This observation suggested that MOP9 was a $\beta$-class PAS protein and that it would form transcriptionally active complexes with CLOCK, MOP4, and the HIF $\alpha$ s.

To determine the expression pattern of MOP9 in human tissues, an adult multiple-tissue Northern blot containing heart, brain, placenta, lung, liver, skeletal muscle, kidney, and pancreas was probed with a fragment of the MOP9 cDNA. This analysis revealed that MOP9 was represented as a relatively lowabundance mRNA that ran as a doublet of $\sim 7.0$ and $8.0 \mathrm{~kb}$ in the brain and placenta, respectively, but was absent in most other tissues (Fig. 2A). A Northern blot containing fetal tissues was also probed, and this blot did not detect MOP9 message in brain, lung, liver, or kidney (data not shown). We also analyzed two Northern blots containing various human brain regions. We found that MOP9 was enriched in the human thalamus, hippocampus, and amygdala but present at lower levels in most other brain regions (Fig. 2A) (Hogenesch et al., 1997; Zhou et al., 1997). Finally, to determine whether MOP9 was expressed in the suprachiasmatic nucleus (SCN), the site of the central circadian oscillator in mammals, we turned to the mouse model and performed in situ hybridization on sections of murine brain. This analysis revealed that the MOP9 message was enriched in the SCN, piriform cortex, and also in the hippocampus of the adult mouse (Fig. $2 B$ ).

To determine whether MOP9 could form functional interactions with circadian and hypoxia factors, transient cell transfection experiments were performed using reporter constructs driven by M34 elements and HREs. The M34 element is a synthetic enhancer generated in our laboratory and has been shown to be similar to elements present in a number of Drosophila and mouse structural genes that are regulated in a circadian manner (Hao et al., 1997; Darlington et al., 1998; Gekakis et al., 1998; Hogenesch et al., 1998). These experiments revealed that MOP9 formed transcriptionally active complexes with CLOCK and its close homolog MOP4, whereas it was incapable of driving expression from the M34 element by itself (Fig. $3 A$ ). In addition, MOP9 appeared to interact more vigorously with CLOCK than did the previously characterized MOP3. Interestingly, MOP9 displayed similar interaction strength as MOP3 with its other partner, MOP4 (Fig. 3A). When cotransfected with HIF1 $\alpha$ and a luciferase reporter construct driven by HREs, MOP9 was able to form a transcriptionally active heterodimeric partnership (Fig. $3 B$ ). In addition, this heterodimeric complex was responsive to cobalt chloride, a mimic of cellular hypoxia (Fig. $3 B$ ). In additional control experiments (data not shown), we observed that the MOP9-Clock dimer is $\sim 10$-fold more active at the M34RE compared with the HRE reporter. In keeping with our previous results, the MOP9-HIF1 $\alpha$ dimer has some degree of affinity for E-box elements and is only approximately twice as active at the HRE compared with the M34RE drive reporter (Hogenesch et al., 1997).

Chromosomal localization of hMOP9 was determined by obtaining a BAC clone and performing FISH. hMOP9 was found to map to chromosome $12 \mathrm{p} 11.22-11.23$, a position near the potassium inward rectifier channel KCNJ8 and AD5, an Alzheimer's disease locus (Inagaki et al., 1995; Pericak-Vance et al., 1997).

\section{DISCUSSION}

Prominent roles in the circadian clock are played by proteins harboring the PAS domain, a signature domain found in proteins that regulate other environmental signaling pathways such as responses to both polycyclic aromatic hydrocarbons and hypoxia. Interestingly, it has been observed that there are commonly multiple mammalian orthologs for each Drosophila member (Hahn, 1998; Taylor and Zhulin, 1999). This observation prompted a search of the GenBank database for a MOP3 paralog. This search uncovered MOP9, a bHLH-PAS orphan with extensive homology to hMOP3 and dCYCLE. Because of this extensive sequence homology, we hypothesized that MOP9 might share similar partners with MOP3 as well as play a role in the regulation of circadian rhythms and also the response to hypoxic stress (Gekakis et al., 1998; Hogenesch et al., 1998).

\section{The circadian response pathway}

Regulation of eukaryotic circadian rhythms involves the use of negative feedback loops and a central circadian oscillator that regulates global rhythms. In Drosophila and mammals, a number of components of this central oscillator have been independently identified by both genetic and biochemical methods (Dunlap, 1999). These components include the transcriptionally active heterodimers (e.g., CLOCK and MOP3), the negative repressors (e.g., PER1, PER2, and PER3) and those proteins involved in facilitating repression (e.g., TIM and DBT) (Dunlap, 1999). The transcriptional activators interact with circadian enhancer sequences present in the structural genes of proteins that are directly regulated by the clock (Hao et al., 1997; Darlington et al., 1998; Gekakis et al., 1998). This enhancer sequence, CACGTGA, is also found in a number of the component genes, such as dTIM, dPER, and mPER clock (Hao et al., 1997; Darlington et al., 1998; Gekakis et al., 1998). Before this report, it was predicted that CLOCK-MOP3 heterodimers upregulate the transcription of the negative repressors producing a feedback inhibitory loop and the generation of circadian oscillation (Darlington et al., 1998). In flies, the blue light photoreceptor CRY can alleviate the repressor activities of the PER and TIM proteins, mediating photic input to the clock and providing a mechanism for light entrainment $(\mathrm{Ce}-$ riani et al., 1999). In mammals, the CRY genes appear to have evolved a different role and are able to repress CLOCK-MOP3 activation directly (Griffin et al., 1999).

In previous work, a role for bHLH-PAS proteins in the maintenance of biological rhythms has been established by multiple methods. In Drosophila, powerful genetics supported by biochemical data have provided compelling proof for a physiological role of CYCLE and CLOCK in regulation of behavioral rhythms (Allada et al., 1998; Darlington et al., 1998; Rutila et al., 1998). However, in mammals, only one screen for circadian phenotypes has been successfully used to reveal a component of the murine clock (Antoch et al., 1997; King et al., 1997). Therefore, our laboratory and others have turned to biochemical methods to 
Figure 3. Interaction of MOP9 with CLOCK and HIF1 $\alpha$. $A$, Interaction of MOP9 with CLOCK and MOP4 in transient transfection experiments. Hep3B cells were cotransfected with $1 \mu \mathrm{g}$ of each expression plasmid and a luciferase reporter driven by three circadian response elements (M34 elements). Relative light units were measured as luciferase units corrected by the $\beta$-galactosidase units as described in Materials and Methods. The data represent the mean $\pm \mathrm{SE}$ of triplicate analyses. $B$, Interaction of MOP9 and ARNT with HIF1 $\alpha$. Hep3B cells were cotransfected with $1 \mu \mathrm{g}$ of each expression plasmid and a luciferase reporter driven by six hypoxia response elements. Relative light units were measured as above, and the data represent the mean $\pm \mathrm{SE}$ of triplicate analyses. Insets, Sequences of the responsive elements used in each assay and denote that a minimal SV40 promoter drives the luciferase reporter.

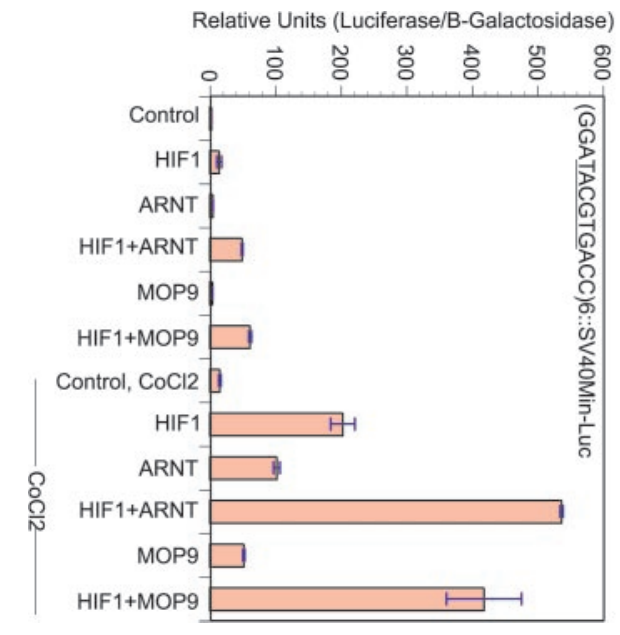

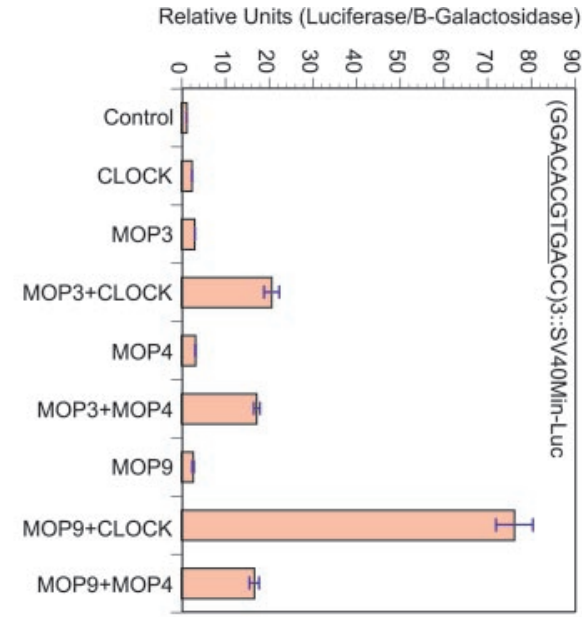

identify and characterize bHLH-PAS proteins that are potentially involved in mammalian circadian rhythmicity. We use three major criteria to predict biologically relevant interactions in such systems. First, we establish pairing rules using in vitro interactions and DNA binding assays. Second, we demonstrate that the predicted bHLH-PAS pair can activate transcription from its cognate response element in a cell culture system. Third, we establish that both members of the predicted $\alpha-\beta$ heterodimer are coexpressed in a tissue or cell type related to the biology under study. This approach led to the original characterization of the CLOCK-MOP3 interaction in mammalian systems and is also the only available proof for a number of other biologically relevant pairs (Hogenesch et al., 1997; Tian et al., 1997).

\section{Biochemical properties of MOP9}

Prompted by the observation of extensive homology between MOP9 and both MOP3 and CYCLE, we tested the idea that MOP9 and MOP3 would have the same biochemical properties. To this end, we used a transient transfection system with a reporter construct driven by M34 elements. The M34 elements have been previously shown to be recognized by both the CLOCK-MOP3 heterodimer, as well as the MOP4-MOP3 heterodimer (Hogenesch et al., 1998). Moreover, this element is similar to the E-box element found in the promoter of the Drosophila PER gene that is required for proper circadian regulation of the PER mRNA (Hao et al., 1997). Using this reporter system, we observed that the CLOCK-MOP9 heterodimer drove transcription almost four times better than did the previously described CLOCK-MOP3 heterodimer. This could be attributable to the possibility that (1) MOP9 interacts more vigorously with CLOCK than does MOP3; (2) MOP9 forms a more transcriptionally active heterodimeric complex with CLOCK than does MOP3; or (3) MOP9 harbors a more potent transactivation domain than does MOP3. To argue against the latter hypothesis, we compared the relative transcriptional strengths of the MOP3MOP4 complex with the MOP9-MOP4 complex. We observed that both MOP3 and MOP9 appeared to form complexes of equivalent transcriptional activity when paired with MOP4. This observation supports the idea that the transactivational potency of MOP9 and MOP3 is similar, and therefore that MOP9 may be an important partner of CLOCK in vivo. In addition to interacting with CLOCK, MOP3 has also been shown to interact with two members of the HIF $\alpha$-class of bHLH-PAS proteins, HIF $1 \alpha$ and HIF2 $\alpha$ (Hogenesch et al., 1998). In support of the idea that MOP9 is functionally similar to MOP3, we observed that MOP9 was also an efficient partner for $\mathrm{HIF} 1 \alpha$, driving transcription as well as HIF1 $\alpha$-ARNT dimers (Fig. 3B).

\section{Tissue-specific expression of MOP9}

As a result of our previous work, we predicted that determining the expression pattern of MOP9 would also be important step in determining which bHLH-PAS proteins were its important physiological partners. Our initial experiments revealed that MOP9 was not highly expressed in fetal tissues but was expressed as two predominant species of 7 and $8 \mathrm{~kb}$ in brain and placenta mRNA derived from adult human tissue sources (Fig. $2 A$ ). Because many bHLH-PAS proteins have specific expression in different brain regions, we performed another Northern analysis on mRNA derived from various adult human brain regions. In this study, MOP9 mRNA appeared to be enriched in the thalamus, hippocampus, and amygdala (Fig. $2 A$ ). This could be because these areas are enriched in neurons or more likely that MOP9 has a specific function that is critical in these tissues. Interestingly, the expression pattern of the predicted $\beta$-class MOP9 overlaps with that of the $\alpha$-class MOP4 in the thalamus. This overlapping expression leads us to propose that the thalamus is a tissue where this partnership has biological relevance.

To explore the brain expression pattern of MOP9 in more detail, in situ analysis was performed using a fragment of the mouse MOP9 cDNA on adult mouse brain sections optimized for the SCN. Low-level MOP9 signal was seen as diffuse staining throughout these brain sections, although enhanced signal was seen in specific sections (Fig. 2). Taken in consideration with the Northern data above, this suggests that MOP9 may be present at lower levels in many neurons, with higher levels in particular brain regions. Importantly, specific MOP9 signal was present in two areas where CLOCK is also expressed, the piriform cortex and SCN (King et al., 1997; Steeves et al., 1999). In addition, specific signal was also seen in the adult hippocampus, a region where CLOCK and MOP4 expression has not been reported. Such an observation is evidence that additional, novel partners for MOP9 exist in these cell populations.

\section{Conclusion}

A number of lines of evidence point to a potential role for MOP9 in circadian gene regulation. First, MOP9 displays extensive sequence identity with MOP3/bMAL1 and CYCLE. CYCLE has been implicated in circadian regulation by genetic and biochemical studies, whereas MOP3 has been demonstrated to be a potential partner for CLOCK in a number of biochemical studies (Gekakis et al., 1998; Hogenesch et al., 1998; Rutila et al., 1998). Second, the brain-specific expression pattern of MOP9 and its presence in the SCN suggests that it has a role in regulation of locomotor activity. Finally, MOP9 is the most potent CLOCK partner described to date, interacting with response elements 
present in structural genes of known clock components. An interesting question that remains unanswered is why multiple CLOCK partners exist in mammals? The observation that circadian rhythmicity of the PER genes occurs in peripheral tissues (and indeed the existence of peripheral clocks) could be related to the evolution of multiple mammalian paralogs of the Drosophila antecedent (Plautz et al., 1997; Zylka et al., 1998). Ultimately, it will take mouse gain and loss of function MOP3 and MOP9 models to discriminate their individual contributions to physiology.

\section{REFERENCES}

Allada R, White NE, So WV, Hall JC, Rosbach M (1998) A mutant Drosophila homolog of mammalian Clock disrupts circadian rhythms and transcription of period and timeless. Cell 93:791-804.

Altschul SF, Gish W, Miller W, Myers EW, Lipman DJ (1990) Basic local alignment search tool. J Mol Biol 215:403-410.

Antoch MP, Song EJ, Chang AM, Vitaterna MH, Zhao Y, Wilsbacher LD, Sangoram AM, King DP, Pinto LH, Takahashi JS (1997) Functional identification of the mouse circadian Clock gene by transgenic BAC rescue. Cell 89:655-667.

Burbach KM, Poland A, Bradfield CA (1992) Cloning of the Ah receptor cDNA reveals a distinctive ligand-activated transcription factor. Proc Natl Acad Sci USA 89:8185-8189.

Carver LA, LaPres JJ, Jain S, Dunham EE, Bradfield CA (1998) Characterization of the Ah receptor-associated protein, ARA9. J Biol Chem 273:33580-22587.

Ceriani MF, Darlington TK, Staknis D, Mas P, Petti AA, Weitz CJ, Kay SA (1999) Light-dependent sequestration of TIMELESS by CRYPTOCHROME. Science 285:553-556.

Chen JD, Li H (1998) Coactivation and corepression in transcriptional regulation by steroid/nuclear hormone receptors. Crit Rev Eukaryot Gene Expr 8:169-190.

Crews ST, Thomas JB, Goodman CS (1988) The Drosophila singleminded gene encodes a nuclear protein with sequence similarity to the per gene product. Cell 52:143-151.

Darlington TK, Wager-Smith K, Ceriani MF, Staknis D, Gekakis N, Steeves TDL, Weitz CJ, Takahashi JS, Kay SA (1998) Closing the circadian loop: CLOCK-induced transcription of its own inhibitors per and tim. Science 280:1599-1603.

Dunlap JC (1999) Molecular bases for circadian clocks. Cell 96:271-290.

Gekakis N, Staknis D, Nguyen HB, Davis FC, Wilsbacher LD, King DP, Takahashi JS, Weitz CJ (1998) Role of the CLOCK protein in the mammalian circadian system. Science 280:1564-1569.

Griffin Jr EA, Staknis D, Weitz C (1999) Light-independent role of CRY1 and CRY2 in the mammalian circadian clock. Science 286:768-771.

Gu YZ, Moran SM, Hogenesch JB, Wartman L, Bradfield CA (1998) Molecular characterization and chromosomal localization of a third alpha-class hypoxia inducible factor subunit, HIF3alpha. Gene Expr 7:205-213.

Gu YZ, Hogenesch JB, Bradfield CA (2000) The PAS superfamily: sensors of environmental and developmental signals. Annu Rev Pharmacol Toxicol 40:519-561.

Hahn ME (1998) The aryl hydrocarbon receptor: a comparative perspective. Comp Biochem Physiol C Pharmacol Toxicol Endocrinol 121:23-53.

Hanstein B, Eckner R, DiRenzo J, Halachmi S, Liu H, Searcy B, Kurokawa R, Brown M (1996) p300 is a component of an estrogen receptor coactivator complex. Proc Natl Acad Sci USA 93:11540-11545.

Hao H, Allen DL, Hardin PE (1997) A circadian enhancer mediates PER-dependent mRNA cycling in Drosophila melanogaster. Mol Cell Biol 17:3687-3693.

Hoffman EC, Reyes H, Chu FF, Sander F, Conley LH, Brooks BA, Hankinson O (1991) Cloning of a factor required for activity of the Ah (dioxin) receptor. Science 252:954-958.
Hogenesch JB, Chan WC, Jackiw VH, Brown RC, Gu YZ, Pray-Grant M, Perdew GH, Bradfield CA (1997) Characterization of a subset of the basic-helix-loop-helix-PAS superfamily that interact with components of the dioxin signaling pathway. J Biol Chem 272:8581-8593.

Hogenesch JB, Gu YZ, Jain S, Bradfield CA (1998) The basic-helixloop-helix-PAS orphan MOP3 forms transcriptionally active complexes with circadian and hypoxia factors. Proc Natl Acad Sci USA 95:5474-5479.

Huang ZJ, Edery I, Rosbach M (1993) PAS is a dimerization domain common to Drosophila period and several transcription factors. Nature 364:259-262.

Inagaki N, Inazawa J, Seino S (1995) cDNA sequence, gene structure, and chromosomal localization of the human ATP-sensitive potassium channel, uKATP-1, gene (KCNJ8). Genomics 30:102-104.

Kadesch T (1993) Consequences of heteromeric interactions among helix-loop-helix proteins. Cell Growth Differ 4:49-55.

King DP, Zhao Y, Sangoram AM, Wilsbacher LD, Tanaka M, Antoch MP, Steeves TD, Vitaterna MH, Kornhauser JM, Lowrey PL, Turek FW, Takahashi JS (1997) Positional cloning of the mouse circadian clock gene. Cell 89:641-653.

Perdew GH, Bradfield CA (1996) Mapping the $90 \mathrm{kDa}$ heat shock protein binding region of the Ah receptor. Biochem Mol Biol Int 39:589-593.

Pericak-Vance MA, Bass MP, Yamaoka LH, Gaskell PC, Scott WK, Terwedow HA, Menold MM, Conneally PM, Small GW, Vance JM, Saunders AM, Roses AD, Haines JL (1997) Complete genomic screen in late-onset familial Alzheimer disease. Evidence for a new locus on chromosome 12. JAMA 278:1237-1241.

Plautz JD, Kaneko M, Hall JC, Kay SA (1997) Independent photoreceptive circadian clocks throughout Drosophila. Science 278:1632-1635.

Pugh CW, O'Rourke JF, Nagao M, Gleadle JM, Ratcliffe PJ (1997) Activation of hypoxia-inducible factor-1: definition of regulatory domains within the alpha subunit. J Biol Chem 272:11205-11214.

Reddy P, Jacquier AC, Abovich N, Peterson G, Rosbash M (1986) The period clock of $D$. melanogaster codes for a proteoglycan. Cell 46:53-61.

Reisz-Porszasz S, Probst MR, Fukunaga BN, Hankinson O (1994) Identification of functional domains of the aryl hydrocarbon receptor nuclear translocator protein (ARNT). Mol Cell Biol 14:6075-6086.

Rutila JE, Suri V, Le M, So WV, Rosbach M, Hall J (1998) CYCLE is a second bHLH-PAS clock protein essential for circadian rhythmicity and transcription of Drosophila period and timeless. Cell 93:805-814.

Sangoram AM, Saez L, Antoch MP, Gekakis N, Staknis D, Whiteley A, Fruechte EM, Vitaterna MH, Shimomura K, King DP, Young MW, Weitz CJ, Takahashi JS (1998) Mammalian circadian autoregulatory loop: a timeless ortholog and mPer1 interact and negatively regulate CLOCK-BMAL1-induced transcription. Neuron 21:1101-1113.

Steeves TD, King DP, Zhao Y, Sangoram AM, Du F, Bowcock AM, Moore RY, Takahashi JS (1999) Molecular cloning and characterization of the human CLOCK gene: expression in the suprachiasmatic nuclei. Genomics 57:189-200.

Taylor BL, Zhulin IB (1999) PAS domains: internal sensors of oxygen, redox potential, and light. Microbiol Mol Biol Rev 63:479-506.

Tian H, McKnight SL, Russell DW (1997) Endothelial PAS domain protein 1 (EPAS1), a transcription factor selectively expressed in endothelial cells. Genes Dev 11:72-82.

Wang GL, Jiang BH, Rue EA, Semenza GL (1995) Hypoxia-inducible factor 1 is a basic-helix-loop-helix-PAS heterodimer regulated by cellular $\mathrm{O}_{2}$ tension. Proc Natl Acad Sci USA 92:5510-5514.

Zhou YD, Barnard M, Tian H, Li X, Ring HZ, Francke U, Shelton J, Richardson J, Russell DW, McKnight SL (1997) Molecular characterization of two mammalian bHLH-PAS domain proteins selectively expressed in the central nervous system. Proc Natl Acad Sci USA 94:713-718.

Zylka MJ, Shearman LP, Weaver DR, Reppert SM (1998) Three period homologs in mammals: differential light responses in the suprachiasmatic circadian clock and oscillating transcripts outside of brain. Neuron 20:1103-1110. 